\title{
Trajectory Bundling for Animated Transitions
}

\author{
Fan Du \\ University of Maryland \\ fan@cs.umd.edu
}

\author{
Nan Cao \\ IBM Research \\ nan.cao@gmail.com
}

\author{
Jian Zhao \\ University of Toronto \\ jianzhao@cs.toronto.edu
}

\author{
Yu-Ru Lin \\ University of Pittsburgh \\ yurulin@pitt.edu
}

\begin{abstract}
Animated transition has been a popular design choice for smoothly switching between different visualization views or layouts, in which movement trajectories are created as cues for tracking objects during location shifting. Tracking moving objects, however, becomes difficult when their movement paths overlap or the number of tracking targets increases. We propose a novel design to facilitate tracking moving objects in animated transitions. Instead of simply animating an object along a straight line, we create "bundled" movement trajectories for a group of objects that have spatial proximity and share similar moving directions. To study the effect of bundled trajectories, we untangle variations due to different aspects of tracking complexity in a comprehensive controlled user study. The results indicate that using bundled trajectories is particularly effective when tracking more targets (six vs. three targets) or when the object movement involves a high degree of occlusion or deformation. Based on the study, we discuss the advantages and limitations of the new technique, as well as provide design implications.
\end{abstract}

\section{Author Keywords}

Animated Transitions; Movement Trajectory Bundling; Multiple Object Tracking; Information Visualization

\section{ACM Classification Keywords}

H.5.1 Multimedia Information Systems: Animations

\section{INTRODUCTION}

Recent advances in information visualization have augmented the capacity of static graphics in understanding various aspects of data. For example, in a two-dimensional scatterplot, allowing users to switch between views with axes corresponding to different pairs of variables can help users capture the data distribution across multiple dimensions [14]. Animated transition, which generates a sequence of images to create visual continuity between changes of objects (e.g., colors, sizes, and locations), has become a popular design choice when switching between different views or layouts [8]. When the animation involves shifting the location of objects, continuous movement along the line between an object's initial and destination positions is shown to create a movement trajectory as cues

Permission to make digital or hard copies of all or part of this work for personal or classroom use is granted without fee provided that copies are not made or distributed for profit or commercial advantage and that copies bear this notice and the full citation on the first page. Copyrights for components of this work owned by others than ACM must be honored. Abstracting with credit is permitted. To copy otherwise, or republish, to post on servers or to redistribute to lists, requires prior specific permission and/or a fee. Request permissions from Permissions@ acm.org.

CHI 2015, April 18-23 2015, Seoul, Republic of Korea.

Copyright is held by the owner/author(s). Publication rights licensed to ACM. ACM 978-1-4503-3145-6/15/04...\$15.00. http://dx.doi.org/10.1145/2702123.2702476

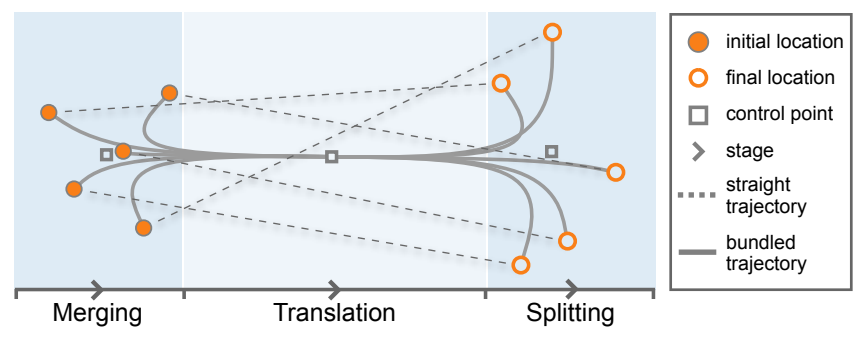

Figure 1. An illustration of the movement trajectories of five objects. Dashed and solid lines represent straight and bundled trajectories.

to help users keep track of the objects and their variations. This simple technique has been commonly used in many situations involving objects' location changes, such as updating the underlying data $[2,8,24]$ or switching between different layout methods [4, 15, 29]. However, when objects' movement paths overlap, or the number of tracking targets grows, tracking moving objects becomes extremely difficult in a typical short period of animated transitions.

There has been prior work studying this issue from different perspectives of animation parameter controls, including staging [5, 14, 21], grouping [14], and timing [6]. Little has been understood about how objects' different movement trajectories affect users' ability of tracking the objects' location changes in a transition. In this paper, we take the initiative to study the effect of movement trajectories, which is a complicated problem involving the following challenges: (1) a variety of choices can be made to generate different objects' movement trajectories, but there is a lack of simple guideline that offers coherent design solutions; (2) users' perceived difficulty of tracking objects can be impacted tremendously not only by the designs of trajectories but also depending on the underlying data distribution, which is difficult to isolate in an experimental study.

In this work, we overcome these challenges by first proposing a simple yet insightful design guideline to facilitate tracking moving objects in animated transitions. Instead of simply moving an object along a straight line, we create "bundled" movement trajectories (as illustrated in Fig. 1) for a group of objects that are close to one another and share similar moving directions. Then, we design a comprehensive controlled user study to test the effect of using bundled trajectories in tracking objects in animated transitions. Using a pilot study and simulations, we decompose the complexity of tracking moving objects into factors, including trajectory lengths and number of targets, and identify three metrics-occlusion, dispersion, and deformation-which measure the tracking task complexity in terms of different spatiotemporal structures of data distribution. We conduct a controlled experiment based on these various complexity aspects. The results indicate 
that tracking moving objects with bundled trajectories leads to a higher tracking accuracy than simple straight trajectories when the number of targets becomes larger (six vs. three targets), or the overall moving occlusion or deformation becomes higher. Based on the study results, we discuss the advantages and disadvantages of the new technique, and provide suggestions for generating movement trajectories to effectively track objects' location changes.

\section{BACKGROUND}

Animation, broadly speaking, creates an illusion of continuous visual changes through displaying a sequence of related static images in a temporal manner. In this paper, we focus on animated transitions, a subset of computer animation techniques to turn abrupt transitions into smooth ones, thus providing a natural user experience, which is widely used in visual system designs in human-computer interaction (HCI) and information visualization (InfoVis) domains. A few general guidelines have been introduced for designing effective animations [20,28], and the advantages and disadvantages of animations have been discussed $[3,28]$. However, many of these recommendations are too general to guide the design of efficient animated transitions, especially in particular applications (e.g., InfoVis) that are concerned in this paper.

Animated transitions can be characterized by two types of parameters: temporal and spatial, i.e., parameters that govern object speed or pacing and ones that involve object trajectories. Some empirical studies about the temporal aspect of animated transitions have been conducted. For instance, Heer and Robertson [14] found that staged animations, i.e., breaking the whole animation into stages of movements, can improve a user's graphical perception for viewing statistical charts. Dragicevic et al. concluded that "slow-in, slow-out" pacing has a better object tracking performance than constant speed in animations [6]. Guilmaine et al. found advantages of using a hierarchical animation technique for tracking certain kinds of changes in tree visualizations [13]. However, such temporal approaches commonly increase the total animation time, which may not optimize users' performance. Moreover, Chevalier et al. discovered that staggering, i.e., delaying the start time of elements incrementally, actually has a negligible effect on object tracking accuracy [5].

The spatial aspect of animated transitions is related to studies on multiple object tracking (MOT) in perceptual psychology [23], since many animations usually require the user to understand which objects (e.g., points, disks, bars, etc.) move to which locations during the transitions. Results from these studies reveal key factors for people's performance in MOT tasks: speed, trajectory, and spacing. Increasing objects' moving speeds impairs tracking accuracy and decreases the maximum number of targets people can track $[1,9,26]$, except for one case when the speed is changed by scaling the display as a whole [10]. Suganuma and Yokosawa found that the tracking accuracy is also impaired when targets share similar trajectories with distractors [27]. Object spacing can be characterized by the concept of crowding. People can track a maximum of 7 or 8 objects when there is adequate spacing, but this number drops as objects become more crowded [10].
Study results also suggest that the identities of targets tend to be lost when they are close to each other and when more distractors are introduced [22]. Despite that extensive research of this topic in psychology literature has provided a lot of empirical data and general guidelines, little has been done to propose techniques and algorithms for improving animated transitions from the spatial aspect (e.g., by carefully planning object trajectories), which is our focus here.

In InfoVis, animated transitions are usually used to smooth the switching between data views, charts, and layouts, which involves location shifting of a large number of objects. Different from general MOT tasks, elements on a visualization view often imply certain relationships among each other, that indicate different "virtual groups" of data items in a user's mental model. For example, points located close-by in a scatterplot share similar attributes, and nearby graph nodes have stronger connections in a force-directed layout [7]. To enhance the understanding and tracing of these relationships during animated transitions, results of the above empirical studies indicate that trajectory is an important cue for perceptual grouping, i.e., letting related targets move in similar trajectories and separate from distractors [27].

For static visualizations, edge bundling [16] is a common technique to reduce the number of line crossings by grouping similar edges, which has been widely applied in many visual representations including graphs, parallel coordinates, and flow maps. Nevertheless, it remains an open problem whether similar techniques can be employed in trajectory bundling in animated transitions to ease a user's tracking relevant targets across two views. Moreover, trajectories in animations have substantial differences from edges in visualizations: (1) trajectories are virtual (not visible), and (2) trajectories consist of both spatial and temporal dimensions (e.g., trajectory crossings may not result in object collisions in an animation). In summary, to the best of our knowledge, this work is the first attempt to enhance animated transitions in the spatial aspect by bundling object trajectories, and study the relevant factors and effect of this spatial approach.

\section{TRAJECTORY BUNDLING}

In this section, we introduce the design rationales and implementation of our trajectory bundling technique, which is proposed for helping users to efficiently track moving objects in animated transitions. We introduce this design based on the findings from several related studies:

- In a static display of objects, people tend to group the objects by their spatial proximity (Gestalt's proximity principle [19]). Similarly, in animated transitions, people tend to perceive objects with similar trajectories as a group [27].

- When tracking multiple objects, people are more likely to perceptually group moving objects together if visual cues of grouping are provided, and such grouping strategy can improve people's tracking performance [30].

- Distorted trajectories have minor impact on tracking single or multiple moving objects $[12,18]$. 


\begin{tabular}{llll}
\hline Factors & Description & $\#$ & $\%$ \\
\hline F1 & Tracking more targets. & 7 & $88 \%$ \\
F2 & Increasing distance between targets. & 7 & $88 \%$ \\
F3 & Increasing number of occlusions. & 5 & $63 \%$ \\
F4 & Tracking over a longer distance. & 4 & $50 \%$ \\
F5 & Frequently changing target group's shape. & 3 & $38 \%$ \\
\hline
\end{tabular}

Table 1. Top factors that increase the difficulty of tracking a group of moving objects reported by the pilot study users.

The first finding suggests that the objects (no matter static or moving) belonging to the same group should be placed close to each other to visually enhance their relationships. The second principle indicates providing visual cues to highlight moving objects' groups is helpful for object tracking.

We designed bundled trajectories (Fig. 1) to incorporate the above design suggestions. More precisely, we used bundled trajectories to smoothly aggregate a group of objects together during an animation, while keeping their initial and final locations unchanged. Such curved trajectories also provide visual cues of objects' group information. The third finding indicates that our design of using curved trajectories could be harmless for object tracking tasks.

We implemented the above design by using B-splines to represent the movement trajectories of a group of objects, which ensures the continuity of the movements. We computed three control points to bundle the splines together. As shown in Fig. 1, these control points are the mean locations of (1) the moving objects' initial positions, (2) the midpoints of their straight movement trajectories, and (3) their final positions. Based on these control points, we separated the transition into three stages: the merging, translating, and splitting of the group of objects. Intuitively, when compared with straight trajectories (dash lines in Fig. 1), bundled trajectories reduce moving collisions between different groups of objects, and show transition trends more clearly.

\section{PILOT STUDY}

We conducted a pilot study (8 participants) to identify factors that affect the difficulty of tracking moving objects and to collect users' feedback on our initial trajectory bundling design. During the study, we randomly generated 16 groups of 3-8 objects on a 2D plane. We ensured the objects in the same group to be closely located and move in similar directions. We showed the objects' movements to our users with either straight or bundled trajectories. We then asked the users to track a selected group of 3 or 6 targets during the animations.

This study helped us to find (1) the key factors that influence users' performance of tracking a group of moving objects and (2) the potential limitation of the proposed technique. Particularly, our pilot users commonly raised five factors as summarized in Table 1, which remarkably increased the tracking difficulty. Based on these factors, we designed a controlled study to evaluate the trajectory bundling technique under different complexity conditions. Also, most users felt the third stage ("splitting") was distracting and difficult to track. To reduce this limitation, we employed the "slow-in, slow-out" strategy in our formal study, to schedule more time for the merging and splitting stages.

\section{STUDY DESIGN RATIONALES}

In this paper, we largely borrowed the study methodologies introduced in [5,6], since we believe the methodologies are not our focus and that reusing the state-of-the-art methods will ensure the accuracy of our experiment. However, it is nontrivial to fit those study methodologies into our problem. Many considerations have been made which will be discussed in this section, including our choices of task, test, testing environment, and complexity metrics of difficulty factors. Particularly, when compared with $[5,6]$, we defined a set of new complexity metrics to capture the features of a group of moving objects instead of individual ones.

\section{Choice of Task}

We focused on evaluating the effectiveness of our trajectory bundling technique at a low perceptual level. Similar with previous studies on animated transitions [5,6], we chose to use visual tracking tasks that require following a set of moving objects (targets), while ignoring other moving objects (distractors). This type of task is fundamental in animated transitions, since high-level tasks are considered to be equivalent to it, or highly depending on it, as discussed in [6].

\section{Choice of Test}

Our study focused on tracking a set of moving objects without identity, which is one type of MOT tasks [5]. At the end of such tasks, to judge the tracking accuracy, participants have to "select all/some" (i.e., select the final locations of all targets $[5,17,25]$ or some of the targets [10]), or "probe one" (i.e., indicate if a particular object belongs to the target set $[9,11,26,30])$, which are two main types of tests.

From the pilot study, we found the first test type does not fit our experiment. In our study, participants had to track up to 6 targets, where selecting all or some of the targets by using a mouse to click on each target dot was tedious and time consuming. Some pilot users complained that they forgot the answers during the selection since it cost too much time.

To avoid this problem, we borrowed the design of the "probe one" test. Particularly, we provided four sets of objects as candidate choices. Participants can review the choices by pressing the space key and make a selection by pressing the enter key; thus avoided inefficient mouse operations and ensured a quick selection. The four choices consisted of one correct answer and three distractors. We generated the distractors by randomly replacing one-third (i.e., 1 for 3-target tasks, 2 for 6-target tasks) of the targets in the correct answer with their nearest neighbors in the final frame. We conducted a series of tests with users after the pilot study to determine the proper replacing ratio that ensured a moderate task difficulty. Similar with the "probe one" test, in our design, the tracking accuracy of a test is 1 if the correct answer is selected, and 0 otherwise.

\section{Choice of Testing Environment}

To ensure participants focus on objects' movements in the study, we represented the objects as visually identical dots with the same shape, color, and size. This avoided the distraction of perceptual visual features, such as pre-attentive colors and shapes. 


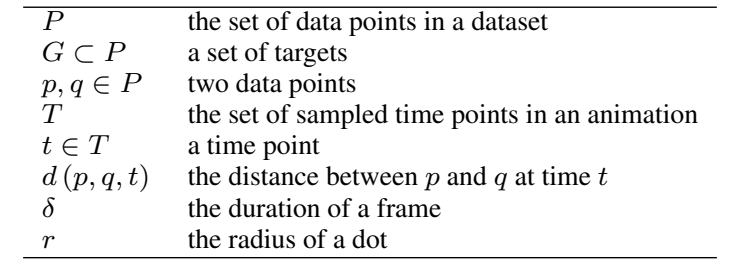

Table 2. Notations used in the definition of the complexity metrics.

Also, we kept the dots relatively small to reduce occlusions during the movements. Here, we chose to control the size of the dots instead of the size of the displaying window for two reasons: (1) the window size should be fixed to ensure a precise control of movement distance, which is a key factor that influences tracking performance [10], and (2) reducing the size of visual items to reduce visual clutter is a common strategy that has been used in many visualization designs with high-density displays, such as node-link diagrams and multidimensional projections.

Finally, we conducted our study with a regular sized (15.4 inches) laptop instead of a big screen because the magnification changes of the display only have minor impact on people's object tracking performance [11].

\section{Choice of Complexity Metrics}

To better understand how the proposed trajectory bundling technique affects users' performance of tracking multiple moving objects under different conditions (such as different trajectory lengths or target numbers), we defined a set of complexity metrics (occlusion, dispersion, and deformation) based on the pilot study results for quantitatively measuring the complexity of these conditions. When compared with the existing metrics introduced in [5,6], our new metrics focus on capturing group features of a set of related moving objects instead of the features of individual ones.

\section{Metrics Definition}

Based on the notations described in Table 2, we define our complexity metrics as follows. An illustration of these metrics is shown in Fig. 2.

Occlusion. Previous studies show that tracking accuracy is impaired when targets occlude with distractors $[1,9]$. To measure the crowding between targets and distractors (i.e., how often targets occlude with distractors), we define a target crowding metric to capture the number of occlusions between targets and distractors during an animated transition:

$$
\operatorname{occlude}(G)=\frac{1}{|T|} \sum_{t \in T} \frac{\sum_{p \in G, q \notin G} \operatorname{overlap}(p, q, t)}{|G|(|P|-|G|)}
$$

where $\operatorname{overlap}(p, q, t)$ is a binary function that determines whether $p$ overlaps with $q$ at time $t$. It returns 1 when an overlap occurs $(d(p, q, t) \leq 2 r)$; otherwise, it returns 0 . $|G|$ is the number of targets, and $(|P|-|G|)$ is the number of distractors outside the target set. $|G|(|P|-|G|)$ normalizes the overall occlusions between targets and distractors at any given time $t$, and thus ensures the metric scores to be independent of the number of targets. At last, we compute the

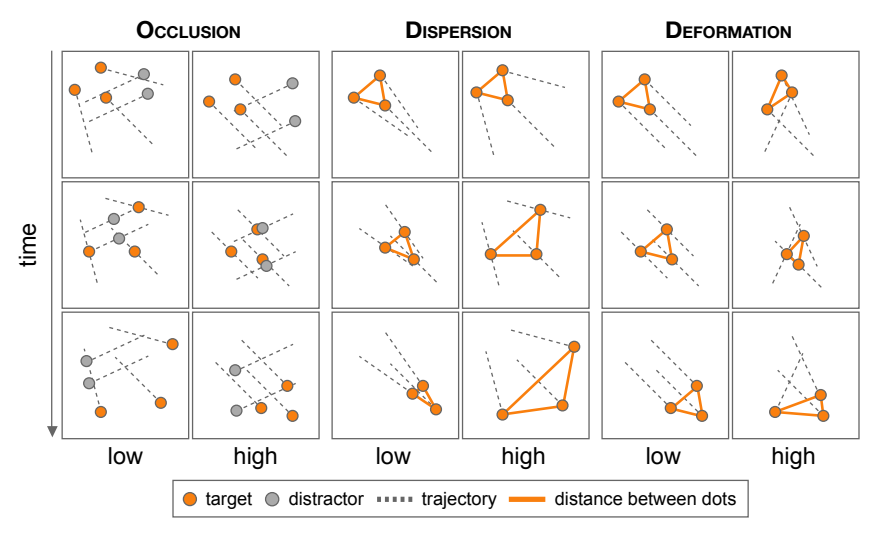

Figure 2. Illustration of the complexity metrics.

mean across all time points during an animation, as the final score of the occlusion metric.

Dispersion. Animation design guidelines proposed by Heer and Robertson [14] suggest grouping similar transitions to help viewers apprehend elements undergoing the same operation. Also, Gestalt's proximity principle [19] reveals that objects near to each other are often perceived as a group. These were supported by our pilot study results. To measure the proximity of targets (i.e., how close they are to each other), we define a target dispersion metric to capture the pairwise distances of the target set during an animated transition:

$$
\text { disperse }(G)=\frac{1}{|T|} \sum_{t \in T} \frac{\sum_{p_{i}, p_{j} \in G, i<j} d\left(p_{i}, p_{j}, t\right)}{\left(\begin{array}{c}
|G| \\
2
\end{array}\right)}
$$

where $|G|$ is the number of targets, and $\left(\begin{array}{c}|G| \\ 2\end{array}\right)$ is the number of 2-combinations of the target set $G$. $\left(\begin{array}{c}|G| \\ 2\end{array}\right)$ normalizes the overall dispersion at any given time $t$, to make the metric scores independent of the number of targets. Finally, we compute the mean across all time points of an animation, as the final score of the dispersion metric.

Deformation. Previous work demonstrates that people's tracking accuracy is improved when targets' virtual shape remains constant [30]. To measure the change in targets' virtual shape (i.e., the difference of the targets' pairwise distance between two consecutive frames), we employed the metric definition proposed by [5], which captures all segments changes of the structure of a group of dots:

$$
\begin{aligned}
& \text { deform }(G)= \\
& \frac{1}{|T|} \sum_{t \in T} \frac{\sum_{p_{i}, p_{j} \in G, i<j}\left|d\left(p_{i}, p_{j}, t\right)-d\left(p_{i}, p_{j}, t-\delta\right)\right|}{\left(\begin{array}{c}
|G| \\
2
\end{array}\right)}
\end{aligned}
$$

where $|G|$ is the number of targets, and $\left(\begin{array}{c}|G| \\ 2\end{array}\right)$ is the number of 2-combination of the set of targets $G$. $\left(\begin{array}{c}|G| \\ 2\end{array}\right)$ normalizes the overall deformation between any given time $t$ and $t-\delta$ ( $\delta$ is the duration of a frame), and thus ensures the metric scores are not influenced by the amount of targets. In the end, we compute the mean across all time points of an animation, as the final score of the deformation metric. 


\section{Analysis and Discussion}

Based on the above definitions, we analyzed the independence of the three complexity metrics via a correlation analysis, and explored the effect of bundled trajectories on the metric scores, when compared with straight trajectories.

Independence analysis. To analyze the independence of the proposed complexity metrics, we randomly generated 10,000 testing datasets. Each dataset contained 16 moving groups, and each moving group consisted of 3-8 moving objects. For each dataset, we randomly selected a set of 6 objects falling within the same group as the targets, and used straight trajectories for the transitions of all the objects. Based on these settings, we computed the scores of the three complexity metrics for each testing dataset, yielding 10,000 scores per metric. We then used these scores to calculate the Pearson correlation coefficients between each two metrics. The analysis results were summarized in Table 3. It shows that the proposed metrics were significantly independent of each other ( $\mathrm{p}$-values $<.001)$, which confirmed their capability of capturing different complexity aspects of animated transitions with straight trajectories.

\begin{tabular}{lc}
\hline Metrics & Correlation $(r)$ \\
\hline occlusion / dispersion & -0.099 \\
occlusion / deformation & -0.093 \\
dispersion / deformation & 0.090 \\
\hline
\end{tabular}

Table 3. The pairwise Pearson correlation coefficients between each of the two complexity metrics. All have p-values less than .001.

Comparison of techniques. We compared straight and bundled trajectories based on the aforementioned complexity metrics, to see how bundled trajectories affect the scores of different metrics. To achieve this goal, we computed another set of complexity scores using bundled trajectories on the above generated testing datasets. We drew these two sets of scores (straight, bundled) in three scatter plots, one for each metric (Fig. 3). There are a total of 10,000 dots in each scatter plot, where each dot represents a testing dataset and its $x$ and $y$ coordinates represent the scores computed using straight and bundled trajectories, respectively. A lower score indicates a lower complexity.

In Fig. 3, we found that overall bundled trajectories reduced the occlusion and dispersion scores, and thus reduced the complexity of animated transitions from these two aspects. However, bundled trajectories increased the deformation scores, which was a reasonable finding, since curves have a higher deformation on average than straight lines. In order to further examine the theoretical results in practice, we designed a user study to compare the straight and bundled trajectories, and investigate the benefits and limitations of the trajectory bundling technique.

\section{USER STUDY}

Based on the aforementioned study design rationales, in this section, we introduce a controlled user study designed for two purposes: (1) to evaluate if the task complexity scores computed based on the three metrics are consistent with task difficulty perceived by users, and (2) to compare the effectiveness between straight and bundled trajectories.

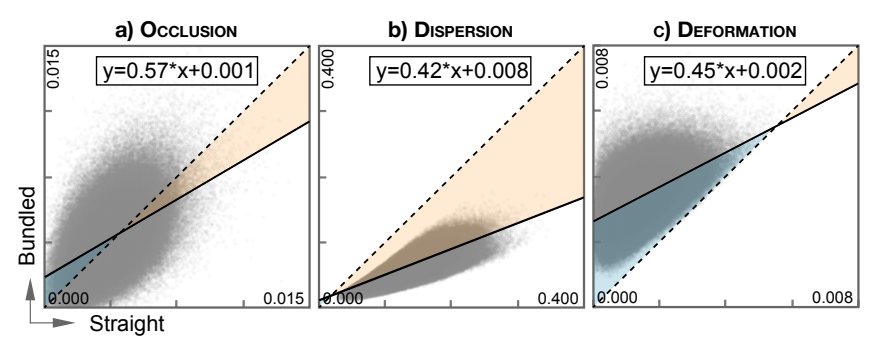

Figure 3. The comparison on computed scores between straight and bundled trajectories under each complexity metric. Predictor functions are annotated, with p-values less than .001. The dashed diagonal lines represent the equivalence of the complexity scores computed using each of the two types of trajectories. The solid lines are the linear regression results. The orange and blue areas show the decreasing and increasing of complexity scores when using bundled trajectories.

\section{Participants and Apparatus}

We recruited 16 volunteers ( 6 female) to participate in our study for comparing the two distinct techniques: animated transitions of moving objects with straight and bundled trajectories. All of the participants had normal vision, and were graduate students studying computer science. Their ages ranged from 23 to 33 (mean 27, $S D=3.16$ ).

The study was performed on a laptop computer with a 15.4inch display of resolution $1440 \times 900$ pixels and $60 \mathrm{~Hz}$ refresh rate. Dots were displayed as circles of 9 pixels $(0.2 \mathrm{~cm})$ in radius, filled with black color. The animation window was $630 \times 630$ pixels $(16.8 \times 16.8 \mathrm{~cm})$ in dimension, with a white background. Participants sat $55 \mathrm{~cm}$ from the display.

\section{Procedure}

At the beginning, we introduced the study to the participants and showed them example animations of two types of tasks: (1) tracking 3 targets moving along straight and bundled trajectories with the length of 0.5 units $^{1}$ and 0.8 units, and (2) tracking 3 and 6 targets moving along straight and bundled trajectories with the length of 0.5 units. These examples helped the participants to get familiar with our study tasks and gain some intuitions about the effect of the trajectory length and the number of targets.

Next, we gave the participants a brief lesson to teach them about using our study system to complete object tracking tasks. Particularly, in each task, users were first showed a set of dots among which targets were highlighted in red and distractors were in black. After memorizing the targets, users had to press the space key to play the animation of a study trial. Half a second before the animation started, the targets changed to black to avoid the distractions of colors. During the animation, users were asked to track the movements of the target dots. When the animation finished, users had to select the final locations of all targets based on four given choices, by pressing the space key to review the choices rotationally and pressing the enter key to make the selection.

Before the formal study, we asked the participants to finish 8 practice tasks using both techniques. Each of these tasks was at different complexity levels and had different trajectory lengths and different numbers of targets. These practice 
tasks were designed to help users get familiar with different task complexities. We encouraged the participants to ask questions during the practice and provided them with the solutions of the practice task to ensure all participants fully understood the tasks and the two types of trajectories.

After the above preparation, we conducted the formal study. In the study, we fully randomized the order of the tasks and counterbalanced the order of using straight and bundled trajectories. For a given task, we used the same dataset with both types of trajectories to ensure a fair comparison. To avoid learning effect, we mirrored and rotated a dataset before reusing it. This approach ensured that for each of the two trajectory types in a task, the participants were tracking the same set of targets using the same dataset, but were unable to memorize the correct answer.

The tracking accuracy was recorded after each trial. After the study, the participants completed a post-study questionnaire to provide their subjective assessment of the difficulty of tasks of different factors (i.e., trajectory type, path length, and target number). We also conducted a brief informal interview to collect feedback from them. The whole study took approximately one hour per participant, during which the participant can take a break when needed.

\section{Experimental Design and Task Generation}

We employed a full-factorial within-subjects study in which each participant had to perform the following 96 study tasks twice, once for each of the two tested trajectory designs (two study blocks), yielding $3,072(16 \times 96 \times 2)$ trials in total.

As summarized in Table 4, the three independent variables that we considered in our task design were: (1) trajectory length ( 0.5 units or 0.8 units), (2) target number ( 3 or 6 ), and (3) task complexity ( 3 metrics $\times 2$ levels).

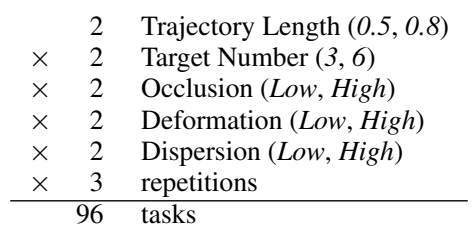

Table 4. The design of study tasks.

We generated the above tasks in two steps. We first randomly computed a large number of transition datasets. Based on these datasets, we then selected the ones satisfying certain complexity requirements (e.g., low occlusion, high dispersion, and low deformation), as a task data used in the study.

Task data generation. We randomly generated 200,000 transition datasets, half of which (100,000 sets) used trajectories of 0.5 units in length and the other half used trajectories of 0.8 units in length (straight-line distance). Each dataset contained 80 moving objects represented as dots with a fixed radius of 0.014 units. We ensured that: (1) no dot overlapping existed at the initial and final frames, to avoid unnecessary confusions in users' target memorizing and answer selecting, and (2) all transitions had an equal straight-line length, to ensure objects' movements stop at the same time.
To generate data for tasks with different number of targets, we computed transition groups for each dataset, i.e., identifying groups of moving objects in which objects are close to each other at the initial frame and share similar moving directions. To achieve this goal, we extracted a feature vector $\left(x_{s}, y_{s}, x_{t}, y_{t}\right)$ for each moving object, where $\left(x_{s}, y_{s}\right)$ and $\left(x_{t}, y_{t}\right)$ represent the $x$ and $y$ coordinates of the object's initial and final locations, respectively. This feature vector captured both the direction and location features of moving objects. We then used the K-means clustering algorithm to identify groups of objects (i.e., moving groups) based on this feature vector. In the implementation, we set $K=16$ to make each group contain 5 objects on average. Finally, in each dataset, we randomly selected a group with exactly 3 or 6 objects as the target group of a study task.

Task selection. We selected the datasets used for study tasks from the randomly generated datasets by considering their complexity conditions. First, we computed three metric scores (occlusion, dispersion, and deformation) for each dataset and categorized each dataset into one of three complexity levels (High, Medium, and Low) based on its metric scores. Then, we sorted these datasets by the score of each metric in a descending order, yielding three ranking lists. In each list, for example, the one computed based on occlusion scores, we considered the first one third datasets as the "High" occlusion (labeled as $H$ ), the last one third as the "Low" occlusion (labeled as $L$ ), and the rest as the "Medium" occlusion (labeled as $M$ ). Thus, the datasets can be marked by these complexity notations. For example, the notation $L M H$ represents the subset of the datasets of low occlusion, medium dispersion, and high deformation.

To obtain more sensitive results in the study, we removed datasets with $M$ labels, and kept those with one of the following eight $\left(2^{3}\right)$ complexity notations: $L L L, H L L, L H L, L L H$, $H H L, H L H, L H H$ and $H H H$. For the study tasks, we randomly selected task data that satisfied this complexity requirement from the random datasets.

Task duration. Objects' moving speed is one key factor for people's tracking performance in MOT tasks $[1,9,26]$. To ensure a fair comparison, we chose to control objects' average moving speed in this study, i.e., 0.5 units per second for both types of trajectories. For transitions with straight trajectories, the durations were $1000 \mathrm{~ms}$ ( 0.5 units $)$ and $1600 \mathrm{~ms}(0.8$ units). For transitions with bundled trajectories, we set the durations based on the average curve length of the target dots to guarantee all dots move synchronously. The average curve lengths of 0.5 and 0.8 units (straight distance) were 0.64 $(S D=0.07)$ and $0.91(S D=0.06)$ units; thus the durations were $1280 \mathrm{~ms}$ and $1820 \mathrm{~ms}$. Finally, we employed the "slow-in, slow-out" on both straight and bundled transitions, which did not affect the duration or the average speed.

Note that the duration of transitions with bundled trajectories was longer than those with straight trajectories. This may potentially bias the experiment results towards bundled trajectories. Studies that control the duration to be equal for both types of trajectories were left as future work. 


\section{Hypotheses}

We made the following hypotheses to test in the study:

H1 For both straight and bundled trajectories, low complexity tasks in general have higher accuracy than high complexity tasks in terms of the three complexity metrics (occlusion, dispersion, and deformation), with the exception that low deformation may not be associated with high accuracy for bundled trajectories.

H2 When the number of targets increases, tracking using bundled trajectories has higher accuracy than using straight trajectories.

H3 When the trajectory length increases, tracking using bundled trajectories has higher accuracy than using straight trajectories.

H4 When task complexity increases in terms of complexity metrics (occlusion, dispersion, and deformation), tracking using bundled trajectories has higher accuracy than using straight trajectories.

Among these hypotheses, $\mathbf{H} \mathbf{1}$ was made based on the design of our complexity metrics, and the observation that straight trajectories were significant predictors of bundled trajectories in terms of the metric scores (Fig. 3). We expected to have the exception in H1, since bundled trajectories increased the overall deformation in object tracking tasks (Fig. 3c). H2 and H3 were based on users' feedback from the pilot study, and H4 was based on the fact that bundled trajectories reduced the overall occlusion and dispersion in object tracking tasks (Fig. 3a,b). We expected the deformation metric to be disconfirmed in $\mathbf{H 4}$, for the same reason as in $\mathbf{H 1}$.

\section{Study Results}

In this section, we describe the results of the above study. First, we validate our three complexity metrics (occlusion, dispersion, and deformation) by analyzing their correlations with users' tracking accuracy. Then, we compare straight and bundled trajectories based on the tracking accuracy of tasks with either of these two types of trajectories.

\section{Validation of Complexity Metrics}

Fig. 4 shows the mean accuracies of tasks with straight (a) and bundled (b) trajectories for each complexity level (low, high) in each metric (occlusion, dispersion, and deformation). Repeated Measures ANOVA was employed to compare the difference in mean accuracy between low and high complexity levels of each metric. Results of the ANOVA tests are annotated on the figure (significant ones at the .05 levels are highlighted with a white background).

We found significant differences in all metrics for both straight and bundled trajectories (except for deformation with bundled trajectories as we expected). Specifically, for each metric, tasks where that metric was low had significantly higher accuracy than tasks where that metric was high. This finding was consistent with H1 and showed that our complexity metrics were highly correlated with the accuracy of the tasks. Therefore, these metrics reflected tasks' actual difficulty perceived by the participants.

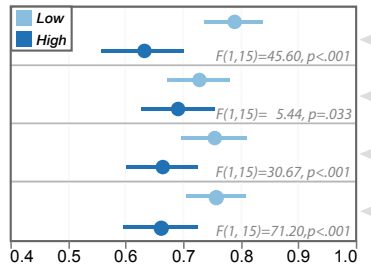

(a) Mean Accuracy (Straight)

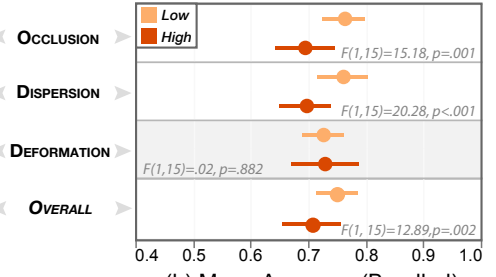

(b) Mean Accuracy (Bundled)
Figure 4. The mean tracking accuracies of tasks with straight (a) and bundled (b) trajectories, when each complexity metric (one per row) was low or high. Error bars are $95 \%$ confidence intervals (CIs).

\section{Comparison between Straight and Bundled Trajectories}

Fig. 5 shows the mean accuracies of 3-target tasks (a) and 6-target tasks (b), with either straight or bundled trajectories, when each complexity metric score was low or high. We conducted Repeated Measures ANOVA tests to compare the mean accuracy between tasks with straight and bundled trajectories, at each level of each metric. Test results are labeled in the figure with significant ones in a white background.

We found that the overall mean accuracy of 6-target tasks with bundled trajectories was significantly higher than with straight trajectories, which supported H2. Also, within 6target tasks, for occlusion and deformation metrics, we observed that the difference of the mean accuracy between bundled trajectory tasks and straight trajectory tasks became larger and significant in high complexity levels, which partially supported H4. Surprisingly, this effect was not found in dispersion metric, which was not supporting H4.

In Fig. 6, the mean accuracies of tasks with 0.5 units (a) and 0.8 units (b) trajectories are presented, with either straight or bundled trajectories for every condition of the three complexity metrics. Repeated Measures ANOVA was conducted to compare the mean accuracy between tasks with straight and bundled trajectories, at each level of each metric (see test results labeled on the figure).

In general, tasks with bundled trajectories had higher accuracy than with straight trajectories, although there was no significant effect for comparing the overall accuracy (Fig. 6). However, significant effect was found in low and high deformation, and low dispersion conditions for 0.8 units length tasks. Moreover, for 0.8 units length tasks, when the occlusion and deformation complexities became higher, the accuracy of tasks with bundled trajectories became better than tasks with straight trajectories, which partially supported H4. Again, this effect was not found in dispersion metric, possibly for the same reason as discussed above.

From Fig. 5a and Fig. 6a, we found that the task accuracy difference between the straight and bundled trajectories techniques did not indicate a trend of increasing or decreasing when the complexities became higher, which seemed not supporting H4. However, these results were aligned with our hypotheses in $\mathbf{H 2}$ and $\mathbf{H 3}$, where the benefits of bundled trajectories would be more obvious when tracking a larger number of targets in longer movement paths. Future studies are warranted to investigate these factors deeper with more levels of target numbers and trajectory lengths. 

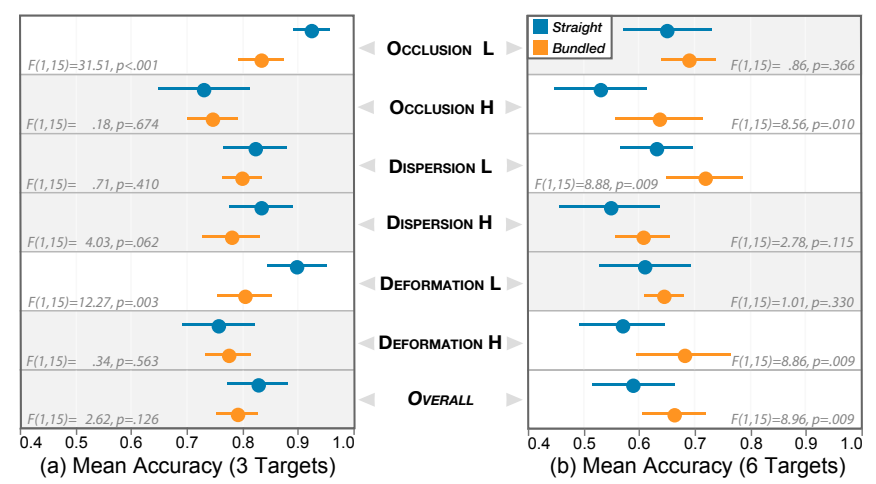

Figure 5. The mean tracking accuracies of 3-target tasks (a) and 6-target tasks (b) with either straight or bundled trajectories, when each complexity metric was low or high. Error bars are $95 \%$ CIs.

\section{Questionnaire}

Our study questionnaire contained eight questions to which the participants responded on a 5-point Likert scale ( $1=$ very easy, $5=$ very difficult). These questions compared the subjective difficulty between tasks with straight and bundled trajectories. Specifically, the comparison was based on tasks with 3 targets (Q1,Q2), 6 targets $(\mathrm{Q} 3, \mathrm{Q} 4), 0.5$ units trajectories (Q5,Q6), and 0.8 units trajectories $(\mathrm{Q} 7, \mathrm{Q} 8)$. The results are shown in Fig. 7.

On average, the participants found tasks with 3 targets were easier than with 6 targets, and tasks with 0.5 units trajectories were easier than with 0.8 units trajectories. These differences were found in tasks with both straight trajectories and bundled trajectories. Comparing straight and bundled trajectories, on average, the participants found for tasks with 6 targets or with 0.8 units trajectories, bundled trajectories were easier to track than straight trajectories. However, for tasks with 3 targets or with 0.5 units trajectories, the participants' assessments of difficulty for the two types of trajectories were nearly the same.

\section{DISCUSSION}

In this section, we first introduce our findings in users' tracking strategies at each stage of animated transitions with bundled trajectories. Then, based on these findings, we explain the experiment results, and discuss the design implications.

\section{Object Tracking Strategies}

In our informal interviews after the study, we showed Fig. 1 to the users and asked them to describe their tracking strategies at each of the three transition stages (merging, translation, and splitting) when using bundled trajectories. We summarized the reported tracking strategies based on the transition stages.

Merging stage. At this stage, objects within a group move towards the first control point and are increasingly merged together. The most popular tracking strategy at this stage was "track one", i.e., following only one target, and then retrieving other targets after they become merged. This strategy reveals that at the merging stage, the movements of objects within a group are consistent and highly predictable; thus only one target has to be precisely tracked, based on which the locations of the rest can be inferred. This finding implies at
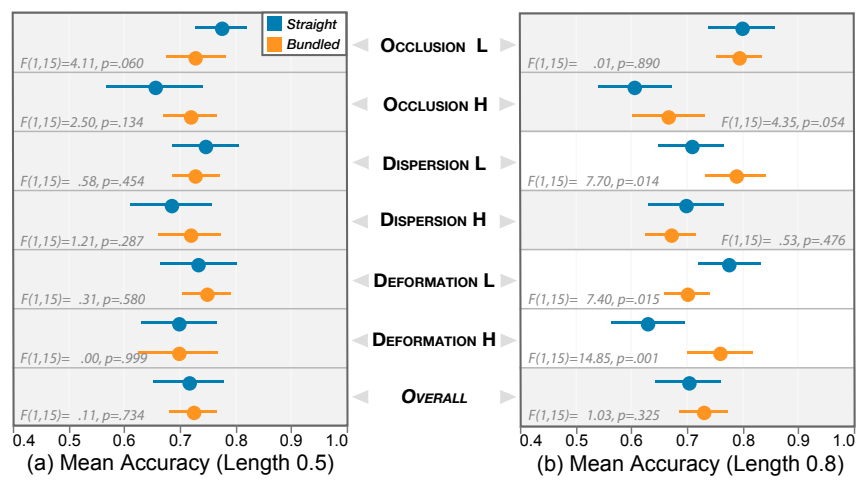

Figure 6. The mean tracking accuracies of 0.5 units trajectory (a) and 0.8 units trajectory (b) tasks, with either straight or bundled trajectories, when each complexity metric was low or high. Error bars are $95 \%$ CIs.

this stage, the occlusions between the targets and distractors, and the deformation of the target group have minor influence on users' tracking accuracy. Moreover, the converging trajectories also reduce the dispersion of the target group, which has a tendency to improve users' tracking accuracy.

Translation stage. At this stage, objects of a group are tightly bundled together and move in a uniform direction. Users commonly developed a "grouping" strategy at this stage, i.e., perceiving and tracking the entire group of multiple objects as one single virtual object. This "grouping" strategy helped reduce the tracking difficulty as reported by our users, and can improve people's tracking accuracy as found in [30]. Moreover, at this stage, since objects within each group were tightly bundled, occlusions between targets and distractors are likely to be reduced, and the dispersion and deformation will also be minimized, thus making it a stage at which moving objects are the easiest to track.

Splitting stage. At this stage, grouped moving objects spread out to different final locations. The users had to track each one of the targets separately, i.e., using a "track all" strategy. During the splitting stage, dispersion and deformation are both increased, and occlusions between targets and distractors would cause more impairment since each target has to be followed precisely. These together make it the most difficult stage for object tracking, as declared by the users.

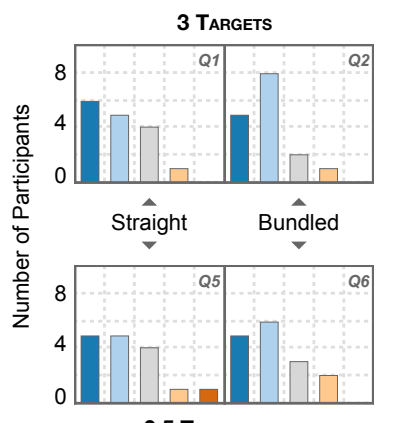

0.5 Trajectories

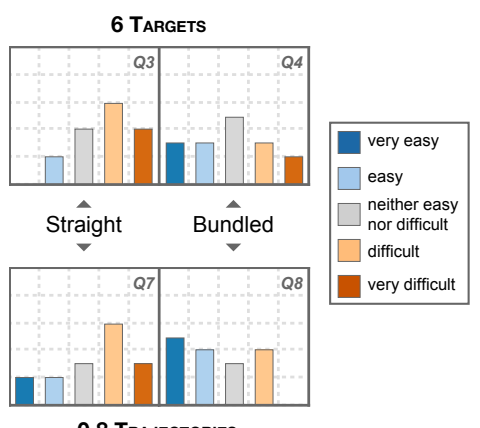

0.8 Trajectories
Figure 7. Results of the Likert-scale (1=very easy, $5=$ very difficult) questions (Q1-Q8) from the questionnaire session. A total of 16 participants responded. 


\section{Exploratory Analysis of Experiment Results}

Based on the experiment results and the users' subjective feedback, we found both advantages and limitations of the proposed trajectory bundling technique. In general, bundled trajectories can help users to:

A1 Track more moving objects at a time. From the experiment, we found that for tasks of tracking 6 targets, using bundled trajectories has a significantly higher overall accuracy than using straight trajectories (Fig. 5b). In the questionnaires, users' subjective assessment of task difficulty also supported this finding (Q3,Q4 in Fig. 7). This benefit could come from the "track one" and "grouping" strategies used in the first two transition stages as discussed above. These two strategies allowed users to only track one moving object (either real or virtual), and thus significantly reduced the tracking difficulty. It implies that at the first two stages of transitions with bundled trajectories, increasing the number of targets only results in minor impairment on users' tracking performance. On the contrary, when using straight trajectories, more objects to track increases the chance of losing targets.

A2 Track moving objects with higher occlusion. In the experiment results, we found that in high occlusion condition, the accuracy of tasks with bundled trajectories was higher than with straight trajectories, and this improvement was significant in 6-target tasks (Fig. 5b). One possible reason is that bundled trajectories can reduce the overall occlusions in object tracking tasks (Fig. 3a), whereas high occlusion increases the chance of losing targets during straight-line movement transitions. Another potential reason is that, by using the "track one" and "grouping" strategies with bundled trajectories, occlusions during the merging and translation stages have less impairment on users' tracking performance, as discussed above.

A3 Track moving objects with higher deformation. From the study results, we found that (1) the tracking accuracy of tasks with bundled trajectories was nearly not impaired by high deformation (Fig. 4b), and (2) in high deformation condition, the accuracy of tasks with bundled trajectories was higher than with straight trajectories, and this improvement was significant in tasks with 6 targets (Fig. 5b) and tasks with 0.8 units trajectories (Fig. 6b). These two findings could be explained by the fact that although high deformation impairs tracking performance at the splitting stage of transitions with bundled trajectories, it has minor influence at the merging and translation stages. The finding (2) implies that this advantage is more remarkable when tracking more objects in long-distance transitions.

From the users' feedback in our informal interviews after the study, we found the major limitation of the trajectory bundling technique was caused by its splitting stage, during which individual targets have to be followed precisely. Specifically, the experiment results indicate that the trajectory bundling technique is less effective in two situations:

L1 Less effective in simple object tracking tasks. In our study, a task is considered to be simple if it satisfies any of the three criteria as follows: (1) the number of targets is small (i.e., 3-target tasks), (2) the trajectory length is short (i.e., 0.5 units trajectory tasks), or (3) any of its complexity metrics (occlusion, dispersion, and deformation) has a low score. The experiment results show simple tasks with straight trajectories achieved similar or higher accuracies than those with bundled trajectories, with only one exception for low dispersion (Fig. 5, Fig. 6). Particularly, straight trajectories significantly outperformed bundled trajectories in 3-target tasks under low occlusion or low deformation condition, and in 0.8 units trajectory tasks under low deformation condition. One explanation is that in the simple tasks, the impairment of the splitting stage outweighed the benefit of the merging and translation stage. This finding indicates the bundling technique is less effective, or even harmful, when the object tracking tasks are too simple.

L2 Less effective in high dispersion condition. Before the study, we hypothesized that in high complexity conditions, tasks with bundled trajectories would have higher accuracy than with straight trajectories (H4). However, the study results for the dispersion metric disconfirmed this hypothesis: bundled trajectories outperformed straight trajectories in low but not in high dispersion tasks (Fig. 5b, Fig. 6b). This could be explained by the observation that high dispersion tended to make the targets more separate at the final frame; thus intensified the weakness of the splitting stage. This finding suggests that in object tracking tasks, the trajectory bundling technique is more helpful when targets are closely distributed in the final frame and is less effective when targets are widely separated.

\section{CONCLUSION AND FUTURE WORK}

We have presented a novel trajectory bundling technique to facilitate tracking moving objects in animated transitions. Specifically, movement trajectories are "bundled" for a group of objects that are close in spatial locations and share similar moving directions. We have described a comprehensive controlled experiment to examine the effect of bundled trajectories by decomposing the complexity of object tracking tasks into different aspects. The experiment results indicate the effectiveness of using bundled trajectories when tracking more targets (six vs. three targets) or when the object movement involves a high degree of occlusion or deformation. Based on the results, we have discussed the strengths and weaknesses of our technique, and outlined design implications for generating movement trajectories to effectively track a group of objects in animated transitions.

Since our study is an initial work in InfoVis and HCI areas to propose and study bundled trajectories in animated transitions, there are a number of promising future directions to pursue. Particularly, our future work will include: (1) study of bundled trajectories in MOT tasks where moving objects have identities; (2) comparison of straight and bundled trajectories when the animation duration is controlled; (3) comparison of the trajectory bundling technique with other visual techniques that help to perceive group information in animated transitions; (4) study of the effect of "slow-in, slow-out" on object tracking with bundled trajectories. 


\section{ACKNOWLEDGMENTS}

This work is partially sponsored by the U.S. Defense Advanced Research Projects Agency (DARPA) under the Social Media in Strategic Communication (SMISC) program (Agreement Number: W911NF-12-C-0028). The views and conclusions contained in this document are those of the authors and should not be interpreted as representing the official policies, either expressed or implied, of DARPA or the U.S. Government.

\section{REFERENCES}

1. Alvarez, G. A., and Franconeri, S. L. How many objects can you track? evidence for a resource-limited attentive tracking mechanism. Journal of Vision 7, 13 (2007), 14.1-10.

2. Bach, B., Pietriga, E., and Fekete, J.-D. Graphdiaries: Animated transitions and temporal navigation for dynamic networks. IEEE TVCG 20, 5 (2014), 740-754.

3. Baecker, R., and Small, I. Animation at the interface, 1990.

4. Cao, N., Gotz, D., Sun, J., and Qu, H. Dicon: Interactive visual analysis of multidimensional clusters. IEEE TVCG 17, 12 (2011), 2581-2590.

5. Chevalier, F., Dragicevic, P., and Franconeri, S. The not-so-staggering effect of staggered animated transitions on visual tracking. IEEE TVCG 20, 12 (2014), 2241-2250.

6. Dragicevic, P., Bezerianos, A., Javed, W., Elmqvist, N., and Fekete, J.-D. Temporal distortion for animated transitions. In Proc. of SIGCHI (2011), 2009-2018.

7. Eades, P. A heuristics for graph drawing. Congr. Numer. 42 (1984), 146-160.

8. Elmqvist, N., Dragicevic, P., and Fekete, J.-D. Rolling the dice: Multidimensional visual exploration using scatterplot matrix navigation. IEEE TVCG 14, 6 (2008), 1539-1148.

9. Feria, C. S. Speed has an effect on multiple-object tracking independently of the number of close encounters between targets and distractors. Attention, Perception \& Psychophysics 75, 1 (2013), 53-67.

10. Franconeri, S. L., Jonathan, S. V., and Scimeca, J. M. Tracking multiple objects is limited only by object spacing, not by speed, time, or capacity. Psychological Science 21, 7 (2010), 920-925.

11. Franconeri, S. L., Lin, J. Y., Pylyshyn, Z. W., Fisher, B., and Enns, J. T. Evidence against a speed limit in multiple-object tracking. Psychonomic Bulletin \& Review 15, 4 (2008), 802-808.

12. Franconeri, S. L., Pylyshyn, Z. W., and Scholl, B. J. A simple proximity heuristic allows tracking of multiple objects through occlusion. Attention, Perception \& Psychophysics 74, 4 (2012), 691-702.

13. Guilmaine, D., Viau, C., and McGuffin, M. J. Hierarchically animated transitions in visualizations of tree structures. In Proc. of AVI (2012), 514-521.
14. Heer, J., and Robertson, G. Animated transitions in statistical data graphics. IEEE TVCG 13, 6 (2007), 1240-1247.

15. Henry, N., Fekete, J.-D., and McGuffin, M. Nodetrix: a hybrid visualization of social networks. IEEE TVCG 13, 6 (2007), 1302-1309.

16. Holten, D. Hierarchical edge bundles: Visualization of adjacency relations in hierarchical data. IEEE TVCG 12, 5 (2006), 741-748.

17. Jardine, N. L., and Seiffert, A. E. Tracking objects that move where they are headed. Attention, Perception \& Psychophysics 73, 7 (2011), 2168-2179.

18. Jolicoeur, P., Ullman, S., and Mackay, M. Curve tracing: A possible basic operation in the perception of spatial relations. Memory \& Cognition 14, 2 (1986), 129-140.

19. Koflka, K. Principles of gestalt psychology. New York: Har (1935).

20. Lasseter, J. Principles of traditional animation applied to 3d computer animation. ACM Siggraph Computer Graphics 21, 4 (1987), 35-44.

21. Plaisant, C., Grosjean, J., and Bederson, B. B. Spacetree: Supporting exploration in large node link tree, design evolution and empirical evaluation. In IEEE InfoVis (2002), 57-64.

22. Pylyshyn, Z. W. Some puzzling findings in multiple object tracking: I. tracking without keeping track of object identities. Visual Cognition 11, 7 (2004), 801-822.

23. Pylyshyn, Z. W., and Storm, R. W. Tracking multiple independent targets: evidence for a parallel tracking mechanism. Spatial Vision 3, 3 (1988), 179-197.

24. Robertson, G., Fernandez, R., Fisher, D., Lee, B., and Stasko, J. Effectiveness of animation in trend visualization. IEEE TVCG 14, 6 (2008), 1325-1332.

25. Scholl, B. J., Pylyshyn, Z. W., and Feldman, J. What is a visual object? evidence from target merging in multiple object tracking. Cognition 80, 1-2 (2001), 159-177.

26. Shim, W. M., Alvarez, G. A., and Jiang, Y. V. Spatial separation between targets constrains maintenance of attention on multiple objects. Psychonomic Bulletin \& Review 15, 2 (2008), 390-397.

27. Suganuma, M., and Yokosawa, K. Grouping and trajectory storage in multiple object tracking: impairments due to common item motions. Perception 35, 4 (2006), 483-495.

28. Tversky, B., Morrison, J. B., and Betrancourt, M. Animation: Can it facilitate? International Journal of Human-Computer Studies 57, 4 (2002), 247-262.

29. Wattenberg, M. Visual exploration of multivariate graphs. In Proc. of SIGCHI (2006), 811-819.

30. Yantis, S. Multielement visual tracking: Attention and perceptual organization. Cognitive Psychology 24, 3 (1992), 295-340. 